\title{
BEST UNIFORM APPROXIMATION BY SOLUTIONS OF ELLIPTIC DIFFERENTIAL EQUATIONS
}

\author{
P. M. GAUTHIER AND D. ZWICK
}

\begin{abstract}
We investigate best uniform approximations to continuous functions on compact subsets of $\mathbb{R}^{n}$ by solutions of elliptic differential equations and, in particular, by harmonic functions. An axiomatic setting general enough to encompass problems of this kind is given, and in this context we extend necessary and sufficient conditions for best harmonic approximation on precompact Jordan domains to arbitrary compact sets and to more general classes of solutions of linear elliptic differential equations.
\end{abstract}

\section{INTRODUCTION}

The subject of harmonic and holomorphic approximation may be viewed as a generalization of classical extension theorems: given a function $f$ with domain $A$, one is interested in knowing whether $f$ may be extended, under preservation of certain smoothness properties, to a superset $B$ of $A$. In many cases where extension is not possible, it suffices to approximate $f$ on $A$, in some convenient norm, by functions defined on $B$ with the desired smoothness. Theorems giving information on the existence of such approximations are commonly referred to as density theorems. Of these, perhaps the best known is C. Runge's famous theorem of 1885, on the approximation of holomorphic functions defined in a neighborhood of a compact set by holomorphic functions defined in a given open superset. When approximation to an arbitrary degree of precision is not possible, it is natural to consider best approximations. Questions of existence, characterization, uniqueness, and degree of approximation then arise.

Although the study of uniform approximation by harmonic and holomorphic functions, which may be said to have originated with Runge's Theorem, has a long and illustrious history, research into best uniform approximation by such function classes is of more recent origin. Best uniform approximation by harmonic function on subsets of $\mathbb{R}^{n}$ was first considered by Burchard [Bu], and then by Hayman, Kershaw, and Lyons [HKL]. In order to summarize their findings, we first introduce the following notation and terminology.

For a real-valued function $f$ defined on a set $E$ we define

$$
\|f\|_{E}=\sup _{x \in E}|f(x)| \text {. }
$$

Received by the editors January 2, 1991 and, in revised form, March 9, 1992.

1991 Mathematics Subject Classification. Primary 41A50, 41A52, 41A65, 35J15, 31B05.

The first author was supported by NSERC (Canada) and FCAR (Québec). The second author was supported in part by an NSF grant to VT-EPSCoR. 
If $\mathscr{F}$ is a family of functions on $E$, we say that $h \in \mathscr{F}$ is a best approximation to $f$ from $\mathscr{F}$ if

$$
\|f-h\|_{E} \leq\|f-u\|_{E} \quad \text { for all } u \in \mathscr{F} .
$$

When $f$ and $h$ are continuous, and $E$ is compact, the two "extremal sets"

$$
K_{+}(f-h)=\left\{x \in E: f(x)-h(x)=+\|f-h\|_{E}\right\}
$$

and

$$
K_{-}(f-h)=\left\{x \in E: f(x)-h(x)=-\|f-h\|_{E}\right\}
$$

play an important role in characterizing $h$ as a best approximation to $f$. Indeed, let $\mathscr{F}$ be a linear subspace of $C(E)$. We shall say that $\mathscr{F}$ separates the two sets $K_{+}$and $K_{-}$if there exists a $u \in \mathscr{F}$ such that $u>0$ on $K_{+}$and $u<0$ on $K_{-}$. We shall agree that $\mathscr{F}$ always separates $K_{+}$and $K_{-}$if one of them is empty.

The well-known Kolmogorov criterion [Si] for best approximation in $C(E)$, $E$ compact, may now be paraphrased as follows:

Kolmogorov's Criterion. Let $\mathscr{F}$ be a linear subspace of $C(E)$ and let $f \in C(E)$ and $h \in \mathscr{F}$ be given. Then $h$ is a best approximation to $f$ from $\mathscr{F}$ if and only if $\mathscr{F}$ does not separate $K_{+}(f-h)$ and $K_{-}(f-h)$.

A function $u$ defined on an open subset $U$ of $\mathbb{R}^{n}$ is called harmonic if it has continuous partial derivatives up to second order and satisfies Laplace's equation in $U$ :

$$
\Delta u=\frac{\partial^{2} u}{\partial x_{1}^{2}}+\cdots+\frac{\partial^{2} u}{\partial x_{n}^{2}}=0 .
$$

If $S$ is any subset of $\mathbb{R}^{n}, H(S)$ denotes the set of functions that are harmonic in a neighborhood of $S$. In particular, if $S$ is open, then $H(S)$ consists of all functions harmonic in $S$. We also set $A(S)=C(S) \cap H\left(S^{\circ}\right)$, the set of functions continuous on $S$ and harmonic in the interior of $S$.

Let $G$ be a bounded domain in $\mathbb{R}^{n}$. For a compact subset $K$ of $\bar{G}$, let $\hat{K}$ be the union of $K$ with all components $V$ of $G \backslash K$ such that $\partial V \subset K$ (see $\S 1$ for the formal definition of $\hat{K}$ ). The results of [Bu] and [HKL] may now be summarized as follows.

Theorem 0.1. Let $G$ be a Jordan domain in $\mathbb{R}^{n}$, and let $f \in C(\bar{G})$ and $h \in A(\bar{G})$ be given. Let $\mathscr{F}$ denote either $A(\bar{G})$ or $H(G)$. Then $h$ is a best approximation to $f$ (with respect to the norm $\|\cdot\|_{\bar{G}}$ resp. $\|\cdot\|_{G}$ ) if and only if $\left(K_{+}(f-h) \hat{)} \cap\left(K_{-}(f-h) \hat{)} \neq \varnothing\right.\right.$. Moreover, if $h$ is a best approximation to $f$ then it is unique in $\mathscr{F}$.

In this paper we wish to generalize Theorem 0.1 to more general subsets of $\mathbb{R}^{n}$ and to other classes of approximating functions. As a first step we note that for harmonic approximation, the proofs of sufficiency and uniqueness given in the papers cited above depend only on the maximum principle, and are therefore applicable to arbitrary bounded domains in $\mathbb{R}^{n}$ (provided that our definition of $\hat{K}$ replaces theirs). However, the proof of necessity depends in an essential way on certain properties of Jordan domains in $\mathbb{R}^{n}, n \geq 2$. In particular, it requires that $G=\bar{G}^{\circ}$ and that for compact sets $K_{1}, K_{2} \subset \bar{G}$ with $\left(K_{1}\right)^{\hat{\imath}} \cap\left(K_{2} \hat{)}=\varnothing\right.$,

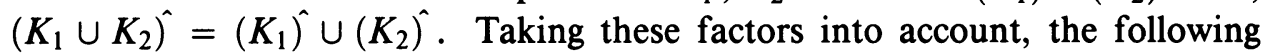
theorem was announced in [Zw]. 
Theorem 0.2. Let $G$ be a bounded domain in $\mathbb{R}^{n}$, let $f \in C(\bar{G})$ and $h \in$ $H(G) \cap C(\bar{G})$ be given, and set $K_{+}=K_{+}(f-h)$ and $K_{-}=K_{-}(f-h)$. If $\left(K_{+}\right) \cap\left(K_{-}\right) \hat{} \neq \varnothing$, then $h$ is the (unique) best harmonic approximation to $f$. Conversely, if $G=\bar{G}^{\circ}$ and $h$ is a best harmonic approximation to $f$, then $\left(K_{+}\right) \hat{n}\left(K_{-}\right)^{\hat{n}} \neq \varnothing$, provided $\left(K_{+} \cup K_{-}\right) \hat{\hat{K}}=\hat{K}_{+} \cup \hat{K}_{-}$.

One approach to proving Theorem 0.2 is to use a combination of a density theorem and a technique known as "translation of poles" or "pole-pushing". In constructive proofs of Runge's theorem in $\mathbb{C}$ (e.g., [SZ]) this technique is used to approximate a rational function on a compact subset $K$ of an open set $G$ to within a certain tolerance by another rational function whose poles lie outside of $G$. It is possible to specify the location of the new poles, provided one can connect each new pole to one of the original poles by an arc in $G \backslash K$. Figuratively, we move each original pole to its new location by pushing it along the appropriate arc. In $\mathbb{R}^{2}$ we may apply this technique to harmonic functions by using the real part of an appropriate holomorphic function. In $\mathbb{R}^{n}, n>2$, a pole-pushing technique described in [GGO] may be applied: We first approximate $g$ by functions with isolated Newtonian singularities and then push the poles into $G^{c}=\mathbb{R}^{n} \backslash G$ as before. When $G=\bar{G}^{\circ}$, we can even push the poles into $\bar{G}^{c}$ to get an approximant that is harmonic in a neighborhood of $\bar{G}$. In particular, it is continuous on $\bar{G}$.

We can now sketch a constructive proof of the necessity part of Theorem 0.2 . Set $K_{ \pm}=K_{ \pm}(f-h)$. Let $K=K_{+} \cup K_{-}$and suppose that $\hat{K}_{+} \cap \hat{K}_{-}=\varnothing$. We define a function $h_{1}$ equal to 1 in a neighborhood of $K_{+}$and equal to -1 in a neighborhood of $K_{-}$. The conditions $\left(K_{+} \cup K_{-}\right)^{\hat{k}}=\hat{K}_{+} \cup \hat{K}_{-}$and $G=\bar{G}^{\circ}$ guarantee that every component of $K^{c}$ meets $\bar{G}^{c}$ (see Lemma 1.3). Thus, we may apply a Runge type theorem with pole-pushing to approximate the harmonic function $h_{1}$ to within $1 / 2$ by a function $h_{2}$ that is harmonic in $G$ and continuous on $\bar{G}$. It follows that $h_{2}$ is positive on $K_{+}$and negative on $K_{-}$, hence by Kolmogorov's criterion $h$ cannot be a best approximation to $f$.

This sketch will provide the outline for similar proofs of necessity in what follows, with the following difference: While an analog to pole-pushing may exist for more general elliptic equations (such as in [DGO]), in place of pole-pushing we shall use nonconstructive density results based on the Lax-Malgrange Theorem. Nevertheless, we have found it illuminating to think of the Runge/LaxMalgrange approximation process in terms of pole-pushing.

We note that a first step in extending the results mentioned above to solutions of elliptic differential equations was made in [HZ]. That work is in the spirit of this paper in that certain properties are isolated that facilitate the characterization of best approximations. But, again, the setting was the closure of a Jordan domain.

In most of what follows, we shall implicitly retain the condition $G=\bar{G}^{\circ}$ in choosing as our setting for the approximation problem an arbitrary compact set $E$, with $E^{\circ}$ playing the role of $G$. In particular, we do not require that $E=$ $\bar{E}^{\circ}$, nor even that $E$ have nonempty interior. However, the approximating class we will consider (functions continuous in a neighborhood of $E$ ) is somewhat more restricted. We return briefly to the setting of functions continuous on $E$ when we present a generalization of Theorem 0.2 in $\S 4$.

In $\S 1$ we abstract certain properties important in harmonic approximation 
and show how they lead to both necessary and sufficient conditions for best approximation in a general setting. We also consider the question of uniqueness. We close $\S 1$ by proving the equivalence of several conditions arising in density theorems. In $\S 2$ and $\S 3$ we give conditions under which solutions of partial and ordinary elliptic differential equations satisfy these properties, and derive as corollaries necessary conditions and sufficient conditions for best approximation from these classes of functions. Section 4 treats the case of harmonic approximation in greater detail. In particular, we generalize Theorem 0.2. Finally, in an appendix we reconcile various versions of the Lax-Malgrange Theorem by proving their equivalence.

\section{AXIOMATIC APPROXIMATION}

In most of this paper, $\Omega$ will denote an arbitrary open subset of $\mathbb{R}^{n}$. However, the results of this section are equally valid if $\Omega$ is any smooth manifold with a countable base [Na2]. A sheaf $\mathscr{H}$ on $\Omega$ of vector spaces is defined by the following stipulations: For every set $A \subset \Omega, \mathscr{H}(A)$ is a vector space whose elements are equivalence classes of real-valued, continuous functions defined in a neighborhood of $A$ (i.e., in an open subset of $\Omega$ containing $A$ ). Two functions that agree in a neighborhood of $A$ belong to the same equivalence class and are considered to be equal in $\mathscr{H}(A)$. Consequently, the sole element of $\mathscr{H}(\varnothing)$ is the zero element. The sum of two elements of $\mathscr{H}(A)$ is realized by adding representative functions in the intersection of their respective domains, which is also a neighborhood of $A$. In general, we will not distinguish between an equivalence class and a function that represents it; for example, we will write $u \in \mathscr{H}(A)$ if $u$ represents an element of $\mathscr{H}(A)$. When necessary, we will write $[u]_{A}$ for the equivalence class of $u$ over $A$. We require further:

(i) If $A$ and $B$ are subsets of $\Omega$ with $A \subset B$ then $\left.\mathscr{H}(B)\right|_{A} \subset \mathscr{H}(A)$ in the sense that if $[u]_{B} \in \mathscr{H}(B)$, then $[u]_{A}$, its "restriction" to $A$, is in $\mathscr{H}(A)$;

(ii) If $A=\bigcup_{\alpha \in I} A_{\alpha}$, where $A_{\alpha}$ are subsets of $\Omega$ and $I$ is an arbitrary index set, and if $u_{\alpha} \in \mathscr{H}\left(A_{\alpha}\right)$ are compatible in the sense that for all $\alpha, \beta \in$ $I, u_{\alpha}=u_{\beta}$ in $\mathscr{H}\left(A_{\alpha} \cap A_{\beta}\right)$ (recall, this means $\left[u_{\alpha}\right]_{A_{\alpha} \cap A_{\beta}}=\left[u_{\beta}\right]_{A_{\alpha} \cap A_{\beta}}$ ), then there is a unique $u \in \mathscr{H}(A)$ such that $u=u_{\alpha}$ in $\mathscr{H}\left(A_{\alpha}\right)$ for each $\alpha$. It follows that if $K_{1}$ and $K_{2}$ are disjoint, compact subsets of $\Omega$, $u_{1} \in \mathscr{H}\left(K_{1}\right)$, and $u_{2} \in \mathscr{H}\left(K_{2}\right)$, then there is a $u \in \mathscr{H}\left(K_{1} \cup K_{2}\right)$ such that $u=u_{1}$ in $\mathscr{H}\left(K_{1}\right)$ and $u=u_{2}$ in $\mathscr{H}\left(K_{2}\right)$.

We wish to introduce several additional properties relative to a sheaf $\mathscr{H}$ on $\Omega$. $\mathscr{H}$ will be said to have the uniqueness property provided that the following condition holds: If $u \in \mathscr{H}(E)$, where $E$ is a connected subset of $\Omega$, and $u=0$ in $\mathscr{H}(V)$ for some open set $V \subset E$, then $u=0$ in $\mathscr{H}(E)$.

We shall say that $\mathscr{H}$ satisfies the strong maximum principle [GT] if the following is true: If $E$ is a subset of $\Omega$ and $u \in \mathscr{H}(E)$, then $u$ is constant in a neighborhood of each point of $E^{\circ}$ (the interior of $E$ relative to $\Omega$ ) at which $u$ has a local maximum (or a local minimum). It follows that $\mathscr{H}$ satisfies the weak maximum principle: If $E$ is a precompact set in $\Omega$ and $\partial E$ denotes its boundary, then

$$
\sup _{E^{\circ}} u \leq \sup _{\partial E} u \quad \text { and } \quad \inf _{E^{\circ}} u \geq \inf _{\partial E} u
$$


Finally, $\mathscr{H}$ will be said to have the local positivity property if for every compact set $K \subset \Omega$ there exists a $u \in \mathscr{H}(K)$ such that $u>0$ in a neighborhood of $K$.

Our interest in such sheaves arises from the fact that the solutions of certain linear elliptic differential equations, of which Laplace's equation is a prototype, form them.

Let $A$ be a subset of $\Omega$. We shall call any component of $\Omega \backslash A$ that is precompact in $\Omega$ a hollow of $A$. An important concept in our theory is that of the hull of a compact set. If $K$ and $G$ are subsets of $\Omega$, with $K$ compact and $G$ open, then we define the hull of $K$ relative to $G$ as the union of $K$ with any hollows of $K$ that are contained in $G$. The hull of $K$ relative to $G$ is denoted by $\hat{K}_{G}$, but we will drop the subscript when no confusion should arise. We will write the hull of a subscripted set, such as $K_{1}$, in the form $\hat{K}_{1}$. We note that our definition differs somewhat from the definitions of $\hat{K}$ given in [Na1, Bu, and HKL]. However, it coincides with them if $G=(\bar{G})^{\circ}$ or if $K$ is a subset of $G$.

We observe that if $K$ is a compact set then $\hat{K}_{G}$ is compact. If $G$ is precompact in $\Omega$ then $\hat{K}_{G}$ is the union of $K$ with all components $V$ of $G \backslash K$ such that $\partial V \subset K$. In any case each hollow of $K$ must have its boundary in $K$.

There is an interesting connection between the hull of a compact set and onepoint compactifications. Let $G^{*}$ denote the one-point compactification of the open set $G$. One can show that if $K$ is a compact subset of $G$ then $K=\hat{K}$ relative to $G$ if and only if $G^{*} \backslash K$ is connected. Indeed, both statements assert that $G \backslash K$ has only nonprecompact components, which are part of a single component in $G^{*} \backslash K$.

Let $f$ be continuous on a compact set $E \subset \Omega$. For a fixed element $h \in$ $\mathscr{H}(E)$ we wish to determine whether $h$ is a best approximation to $f$. Let $K_{+}=K_{+}(f-h)$ and $K_{-}=K_{-}(f-h)$ be defined as subsets of $E$, and let their hulls be taken relative to $E^{\circ}$. Consider the following condition:

$$
\hat{K}_{+} \cap \hat{K}_{-} \neq \varnothing .
$$

Condition (1) may be viewed as a generalization of the well-known Chebyshev alternation criterion for best uniform approximation by linear polynomials on a compact interval [Ch]. Indeed, (1) says that either " $K_{+}$surrounds a point of $K_{-}$" or " $K_{-}$surrounds a point of $K_{+}$."

Theorem 1.1 (Sufficiency). Suppose that $\mathscr{H}$ is a sheaf of continuous functions on $\Omega$ satisfying the weak maximum principle. Suppose that $E$ is a compact subset of $\Omega, f$ is a continuous function on $E, h \in \mathscr{H}(E)$, and (1) holds for $f$ and $h$. Then $h$ is a best approximation to $f$ from $\mathscr{H}(E)$.

Proof. The proof of the sufficiency of (1) is standard (see [Bu and HKL]). Indeed, suppose that $h$ is not a best approximation to $f$ from $\mathscr{H}(E)$; in other words, for some $g \in \mathscr{H}(E)$

$$
\|f-g\|_{E}<\|f-h\|_{E} .
$$

Then on $K_{+}$,

$$
f-g<\|f-h\|_{E}=f-h
$$

and on $K_{-}$,

$$
f-g>-\|f-h\|_{E}=f-h .
$$


If $x \in \hat{K}_{+}$, then $x$ is either in $K_{+}$, in which case $(g-h)(x)>0$, or $x$ is in some component $V$ of $E^{\circ} \backslash K_{+}$with $\partial V \subset K_{+}$. By the weak maximum principle and the compactness of $\partial V, g-h>0$ in $V$. It follows that $g-h>0$ in all of $\hat{K}_{+}$. Similarly, $g-h<0$ in $\hat{K}_{-}$and, consequently, (1) cannot hold.

Theorem 1.2 (Uniqueness). Suppose that $\mathscr{H}$ is a sheaf on $\Omega$ of continuous functions and $E$ is a compact subset of $\Omega$. Suppose that $f$ is a continuous function on $E, h \in \mathscr{H}(E)$, and that (1) holds for $f$ and $h$. If $E^{\circ}=\varnothing$ then $h \equiv f$ on $E$. If $E^{\circ} \neq \varnothing, E$ is connected, and $\mathscr{H}$ satisfies the uniqueness property and the strong maximum principle, then $h$ is the unique best approximation from $\mathscr{H}(E)$.

Proof. If $E^{\circ}=\varnothing$ then (1) implies that $K_{+} \cap K_{-} \neq \varnothing$. In this case $\|f-h\|_{E}=0$; i.e., $f \equiv h$ on $E$.

Now suppose that $E^{\circ} \neq \varnothing$. If $\mathscr{H}$ satisfies the strong maximum principle then it satisfies the weak maximum principle; hence by Theorem 1.1, if (1) is satisfied, then $h$ is a best approximation to $f$ from $\mathscr{H}(E)$. For any $g \in \mathscr{H}(E)$ such that

$$
\|f-h\|_{E}=\|f-g\|_{E},
$$

the same reasoning as in the proof of Theorem 1.1 gives $g \geq h$ on $\hat{K}_{+}$and $g \leq h$ on $\hat{K}_{-}$. If $K_{+} \cap K_{-}=\varnothing$ then (1) implies that either some component $V_{+}$ of $E^{\circ} \backslash K_{+}$with $\partial V_{+} \subset K_{+}$meets $\hat{K}_{-}$at a point $x_{0}$, or else some component $V_{-}$of $E^{\circ} \backslash K_{-}$with $\partial V_{-} \subset K_{-}$meets $\hat{K}_{+}$at a point $x_{0}$. In the first case, $g \geq h$ in $V_{+}$and $g\left(x_{0}\right) \leq h\left(x_{0}\right)$. By the strong maximum principle $g \equiv h$ in a neighborhood of $x_{0}$, hence by the uniqueness property $g=h$ in $\mathscr{H}(E)$. We arrive at the same conclusion if we assume that some component $V_{-}$of $E^{\circ} \backslash K_{-}$with $\partial V_{-} \subset K_{-}$meets $\hat{K}_{+}$. In either case, it follows that $h$ is the unique best approximation to $f$ from $\mathscr{H}(E)$.

If, on the other hand, $K_{+} \cap K_{-} \neq \varnothing$, then $\|f-h\|_{E}=0$, so that $f \equiv h$ on $E$. If $g \in \mathscr{H}(E)$ is any other best approximation then $\|f-g\|_{E}=0$, so $g \equiv h$ on $E$. If $E^{\circ} \neq \varnothing$ and $E$ is connected, by the uniqueness property $g=h$ in $\mathscr{H}(E)$.

Theorem 1.4 will be a partial converse to Theorem 1.1. For its proof we need the following topological lemma.

Lemma 1.3. Let $E$ be any subset of $\Omega$ and let $K \subset E$ be compact. Then the following are equivalent:

(i) $K=\hat{K}$ relative to $E^{\circ}$;

(ii) Every hollow of $K$ meets $\Omega \backslash E$;

(iii) Every hollow of $K$ contains a hollow of $E$.

Remark. Thus, if $E$ has no hollows then $K=\hat{K}$ relative to $E^{\circ}$ if and only if $K$ has no hollows.

Proof of Lemma 1.3. Suppose that $K=\hat{K}$ relative to $E^{\circ}$, and let $V$ be a hollow of $K$. If $V \cap(\Omega \backslash E)=\varnothing$, then $V \subset E$, hence, $V$ being open, $V \subset E^{\circ}$, a contradiction. Therefore, (i) $\Rightarrow$ (ii).

Now assume (ii) and let $V$ be any hollow of $K$. Then there is a point $a \in V \cap(\Omega \backslash E)$. Let $C$ be the component of $\Omega \backslash E$ containing $a$. Since $\Omega \backslash E \subset \Omega \backslash K, C \subset \Omega \backslash K$, while $\partial V \subset K$. If $C$ is not contained in $V$ then 
$C \cap(\Omega \backslash V) \neq \varnothing$ (and $C \cap V \neq \varnothing$ since it contains $a)$. Since $C \cap \partial V=\varnothing$, $C \cap(\Omega \backslash V)=C \cap(\Omega \backslash \bar{V})$, hence $C$ is the disjoint union of two relatively open nonempty sets, a contradiction. Thus, $C$ is a hollow of $E$ contained in $V$, and (iii) is proved. It is immediate that (iii) implies (i).

Remark. If $E$ is compact then we may replace "hollow of $K$ " in part (ii) of Lemma 1.3 by "component of $\Omega \backslash K$."

In order to establish a necessary condition for best approximation we need to assume that $\mathscr{H}$ has one additional property. $\mathscr{H}$ will be said to have the Runge property if whenever $K$ is a compact subset of an arbitrary subset $E$ of $\Omega$, and each hollow of $K$ meets $\Omega \backslash E$, then every function in $\mathscr{H}(K)$ is the uniform limit on $K$ of functions in $\mathscr{H}(E)$.

Theorem 1.4 (Necessity). Suppose that $\mathscr{H}$ is a sheaf of continuous functions on $\Omega$ with the Runge property and the local positivity property. Let $E$ be a compact subset of $\Omega$ and let $f$ be a continuous function on $E$. If $h$ is a best approximation to $f$ from $\mathscr{H}(E)$ then (1) holds, provided that

$$
\hat{K}_{+} \cup \hat{K}_{-}=\left(K_{+} \cup K_{-}\right) \text {. }
$$

Proof. Let (2) hold and suppose that (1) fails, i.e.,

$$
\hat{K}_{+} \cap \hat{K}_{-}=\varnothing \text {. }
$$

We shall show that $\mathscr{H}(E)$ separates $K_{+}$and $K_{-}$which, according to Kolmogorov's criterion, implies that $h$ cannot be a best approximation. Set $K=$ $\hat{K}_{+} \cup \hat{K}_{-}$. By the local positivity property there is a $g_{1} \in \mathscr{H}\left(\hat{K}_{+}\right)$such that $g_{1}>0$ on $\hat{K}_{+}$and a $g_{2} \in \mathscr{H}\left(\hat{K}_{-}\right)$such that $g_{2}>0$ on $\hat{K}_{-}$. Hence, there is a function $g \in \mathscr{H}(K)$ that is positive on $\hat{K}_{+}$and negative on $\hat{K}_{-}$. By compactness, there is a $\delta>0$ such that $g \geq \delta$ on $\hat{K}_{+}$and $g \leq-\delta$ on $\hat{K}_{-}$. Since $K=\hat{K}$, by Lemma 1.3 every hollow of $K$ meets $\Omega \backslash E$. We now use the Runge property to get $u \in \mathscr{H}(E)$ such that

$$
\|u-g\|_{K}<\frac{\delta}{2} .
$$

Now $u>0$ on $\hat{K}_{+}$and $u<0$ on $\hat{K}_{-}$, hence $h$ is not a best approximation to $f$ from $\mathscr{H}(E)$.

Theorem 1.5. Under the conditions of Theorem 1.4, if $E^{\circ}=\varnothing$ then the only functions in $C(E)$ with best approximations from $\mathscr{H}(E)$ are those that can be extended as elements of $\mathscr{H}(E)$.

Proof. Let $f \in C(E)$ and $h \in \mathscr{H}(E)$ be given. Clearly, if $h \equiv f$ on $E$ then $h$ is a best approximation to $f$. Conversely, suppose that $h$ is a best approximation to $f$. Note that for all compact sets $K \subset E, \hat{K}=K$. This is true, in particular, for $K_{+}, K_{-}$, and $K_{+} \cup K_{-}$. We claim that $K_{+} \cap K_{-} \neq \varnothing$. Indeed, if $K_{+} \cap K_{-}=\varnothing$ then the argument in Theorem 1.4 shows that there is a $u \in \mathscr{H}(E)$ such that $u>0$ on $K_{+}$and $u<0$ on $K_{-}$, so that $h$ is not a best approximation to $f$. But if $K_{+} \cap K_{-} \neq \varnothing$ then $\|f-h\|_{E}=0$ and, thus, $h \equiv f$ on $E$. 
In [Wi], J. M. Wilson has constructed an approximation problem showing that, in the absence of condition (2), (1) need not hold in Theorem 1.4. Hence, we have not given a complete characterization of best approximations from $\mathscr{H}(E)$.

We close this section by proving the equivalence of the Runge property to two other properties often encountered in the study of partial differential equations.

Let us say that a sheaf $\mathscr{H}$ on $\Omega$ has the Lax-Malgrange property if for any open sets $U \subset V$ contained in $\Omega$, if $V \backslash U$ has no compact components, then any function in $\mathscr{H}(U)$ may be uniformly approximated on compact subsets of $U$ by restrictions of functions from $\mathscr{H}(V)$. The Lax-Malgrange property plays an important role in the theory of elliptic partial differential equations [Hö] (see also Appendix).

Theorem 1.6. If $\mathscr{H}$ is a sheaf of continuous functions on $\Omega$ then the following conditions are equivalent.

(a) $\mathscr{H}$ has the Runge property;

(b) $\mathscr{H}$ has the Lax-Malgrange property;

(c) if $K$ is a compact subset of an open set $V \subset \Omega$ with $K=\hat{K}_{V}$, and $g \in \mathscr{H}(K)$, then $g$ is the uniform limit on $K$ of elements of $\mathscr{H}(V)$.

Proof. (a) $\Rightarrow$ (b): Let $U \subset V$ be open sets such that $V \backslash U$ has no compact components, and let $K$ be a compact subset of $U$. Set $L=\hat{K}_{U}$; then $L \subset U$ and, from Lemma 1.3, every hollow of $L$ contains a hollow of $U$. But since any hollow of $U$ is compact and $V \backslash U$ has no compact components, it follows that every hollow of $L$ meets $\Omega \backslash V$. If $g \in \mathscr{H}(U)$ then $g \in \mathscr{H}(L)$, where $L=\hat{L}$, hence by (a) $g$ is the uniform limit on $L$ of elements of $\mathscr{H}(V)$. Since $K \subset L$ this holds for $K$ as well, and therefore (b) follows from (a). This direction is based on the proof of [Na1, Theorem 2, p. 113].

(b) $\Rightarrow($ c) : Let $K$ be a compact subset of the open set $V \subset \Omega$ such that $K=\hat{K}$ relative to $V$, and let $g \in \mathscr{H}(K)$. Then there is an open set $W \subset \Omega$ such that $K \subset W$ and $g \in \mathscr{H}(W)$. Moreover, we may assume that $W \subset V$. Since $K=\hat{K}$ relative to $V$, by [Na2, Proposition 3.10.6] $K$ has a fundamental system of neighborhoods $U$ such that $V \backslash U$ has no compact components. Thus, there is an open set $U \subset W$ such that $K \subset U$ and $V \backslash U$ has no compact components. Since $g \in \mathscr{H}(U)$, by (b) $g$ is the uniform limit on $K$ of elements of $\mathscr{H}(V)$. This implication is essentially Corollary 3.10.9 in [Na2].

(c) $\Rightarrow$ (a) : Let $K$ be a compact subset of a set $E \subset \Omega$ such that each hollow of $K$ meets $\Omega \backslash E$, and let $g$ be an element of $\mathscr{H}(K)$. Then $g \in \mathscr{H}(V)$ for some open set $V \subset \Omega$. Let $W$ be a smoothly bounded open set such that $K \subset W \subset \bar{W} \subset V$; then $g$ is in $\mathscr{H}(\bar{W})$. Since $W$ is smoothly bounded, $\bar{W}$ has finitely many hollows, so we may assume (by making $W$ a little smaller, if necessary) that each hollow of $\bar{W}$ is contained in a hollow of $K$. Moreover, since each hollow of $K$ meets $\Omega \backslash E$, we may also assume that each hollow of $\bar{W}$ meets $\Omega \backslash E$. Choose a point of $\Omega \backslash E$ from each hollow of $\bar{W}$, and let $A$ be the set of these (finitely many) points. Set $\Omega^{\prime}=\Omega \backslash A$. By construction, $\bar{W}=(\bar{W})^{\wedge}$ relative to $\Omega^{\prime}$; hence by (c) we can approximate $g$ uniformly on $K$ by elements of $\mathscr{H}\left(\Omega^{\prime}\right)$. Since $E \subset \Omega^{\prime}$, such functions are elements of $\mathscr{H}(E)$. Thus, $\mathscr{H}$ has the Runge property. 


\section{PARTIAL differential equations}

Let $\alpha=\left(\alpha_{1}, \ldots, \alpha_{n}\right) \in \mathbb{N}^{n}, n \geq 2$, be a multi-index, and set $|\alpha|=\alpha_{1}+$ $\cdots+\alpha_{n}$. P will denote a linear partial differential operator on $\Omega \subset \mathbb{R}^{n}$ of the form

$$
P\left(x, \frac{\partial}{\partial x}\right)=\sum_{|\alpha| \leq m} a_{\alpha}(x) \frac{\partial^{\alpha}}{\partial x^{\alpha}}
$$

where

$$
\frac{\partial^{\alpha}}{\partial x^{\alpha}}=\frac{\partial^{|\alpha|}}{\partial x_{1}^{\alpha_{1}} \cdots \partial x_{n}^{\alpha_{n}}},
$$

the coefficients $a_{\alpha}$ are real-valued continuous functions on $\Omega$, and $m \in \mathbb{N}$ is the order of the operator. If $U$ is an open subset of $\Omega$, we denote by $\mathscr{H}(U)$ the space of real solutions of the equation $P u=0$ in $U$; that is, the space of functions $u$ that are $m$-times continuously differentiable in $U$ and satisfy

$$
\sum_{|\alpha| \leq m} a_{\alpha}(x) \frac{\partial^{\alpha} u}{\partial x^{\alpha}}(x)=0, \quad x \in U
$$

If $A$ is an arbitrary subset of $\Omega$, then $\mathscr{H}(A)$ denotes the set of continuous functions that are solutions of $P u=0$ in a neighborhood of $A$. For a fixed operator $P$, this defines a sheaf $\mathscr{H}$ on $\Omega$.

The operator $P$ is said to be elliptic on $\Omega$ if

$$
\sum_{|\alpha|=m} a_{\alpha}(x) \xi^{\alpha} \neq 0
$$

for all $x \in \Omega$ and all nonzero $\xi \in \mathbb{R}^{n}$.

Regularity Theorem. If $P$ is elliptic on $\Omega$, with analytic coefficients, then solutions of $P u=0$ are analytic, and hence the sheaf $\mathscr{H}$ of solutions of $P u=0$ has the uniqueness property.

In [GL] weaker conditions implying the uniqueness property are given. Specifically, if $P$ is a second order uniformly elliptic operator in divergence form with Lipschitz continuous coefficients, and $n \geq 3$, then the solutions of $P u=0$ have the uniqueness property. The uniqueness property for solutions of $P u=0$, where $P$ is a second order elliptic operator with $C^{1}$ coefficients, is proved in $[\mathrm{Ag}]$.

Lax-Malgrange Theorem $[\mathrm{Na} 2,3.10 .7 ; 3.10 .10]$. Let $U \subset V$ be open sets in $\mathbb{R}^{n}$ and let $P$ be an elliptic operator with real analytic coefficients on $V$. Then any solution of $P u=0$ in $U$ may be uniformly approximated on compact subsets of $U$ by restrictions of solutions to $P v=0$ in $V$ if and only if $V \backslash U$ has no compact components.

It follows that if $P$ is an elliptic operator with analytic coefficients on an open set $\Omega$ in $\mathbb{R}^{n}$ then the sheaf $\mathscr{H}$ on $\Omega$ of solutions of $P u=0$ has the Runge property.

Strong Maximum Principle [GT]. Suppose that

$$
P\left(x, \frac{\partial}{\partial x}\right)=\sum_{i, j=1}^{n} a_{i j}(x) \frac{\partial^{2}}{\partial x_{i} \partial x_{j}}+\sum_{k=1}^{n} b_{k}(x) \frac{\partial}{\partial x_{k}}
$$


is elliptic on $\Omega, a_{i j}=a_{j i}$. If the coefficients $a_{i j}$ and $b_{k}$ are continuous in $\Omega$ then the sheaf $\mathscr{H}$ on $\Omega$ of solutions of $P u=0$ satisfies the strong maximum principle.

Combining the results of $\S 1$ and $\S 2$, we have

Theorem 2.1 (Sufficiency). Let $P$ be a second order elliptic operator on $\Omega$ with continuous coefficients, given by (4), and let $\mathscr{H}$ be the sheaf on $\Omega$ of solutions of $P u=0$. Suppose that $E$ is a compact subset of $\Omega, f \in C(E), h \in \mathscr{H}(E)$, and (1) holds for $f$ and $h$. Then $h$ is a best approximation to $f$ from $\mathscr{H}(E)$.

Theorem 2.2 (Uniqueness). Let $P$ be a second order elliptic operator on $\Omega$ with $C^{1}$ coefficients, given by (4), and let $\mathscr{H}$ be the sheaf on $\Omega$ of solutions of $P u=0$. Let $E$ be a compact subset of $\Omega, f$ a continuous function on $E$, $h \in \mathscr{H}(E)$, and suppose that (1) holds for $f$ and $h$. If $E^{\circ}=\varnothing$ then $h \equiv f$ on $E$. If $E^{\circ} \neq \varnothing$ and $E$ is connected, then $h$ is the unique best approximation to $f$ from $\mathscr{H}(E)$.

Theorem 2.3 (Necessity). Suppose that $P$ is an elliptic operator on $\Omega$ having analytic coefficients and locally positive solutions. Suppose that $E$ is a compact subset of $\Omega$ and $f$ is a continuous function on $E$. If $h$ is a best approximation to $f$ from $\mathscr{H}(E)$ then (1) holds, provided (2) is valid.

\section{ORDINARY DIFFERENTIAL EQUATIONS}

Consider the linear differential equation

$$
P u=a_{m}(x) \frac{d^{m} u}{d x^{m}}+\cdots+a_{0}(x) u=q(x),
$$

where the functions $a_{m}, \ldots, a_{0}, q$ are continuous on an open interval $\Omega \subset \mathbb{R}^{1}$. In terms of our previous definition, it is natural to say that the differential operator $P$ is elliptic on $\Omega$ if $a_{m}(x) \neq 0$ for all $x \in \Omega$. The study of elliptic operators is thus equivalent to the study of operators with leading coefficient equal to one.

If $n>1$ and the coefficients of the elliptic operator $P$ are analytic on an open set $U \subset \mathbb{R}^{n}$, then the Lax-Malgrange Theorem implies that "most" solutions of $P u=0$ on $U$ do not extend continuously to any point of $\partial U$ even though $P$ may be defined in a larger open set $\Omega$. Indeed, the space $\mathscr{H}(U)$, endowed with the topology of uniform convergence on compact subsets, is of second category and the subfamily $\mathscr{F}$ of solutions which do not extend continuously to any point of $\partial U$ is residual; that is, $\mathscr{H}(U) \backslash \mathscr{F}$ is of first category. We now sketch a proof of this assertion.

Let $\left\{p_{i}\right\}$ be a countable dense subset of $\partial U$ and for $j=1,2, \ldots$ set

$$
G_{i j}=\left\{u \in H(U):|u(x)| \leq j \text { for } x \in U,\left|x-p_{i}\right| \leq 1 / j\right\} .
$$

Then

$$
H(U) \backslash \mathscr{F} \subset \bigcup_{i, j=1}^{\infty} G_{i j},
$$

and since each $G_{i j}$ is clearly closed, it is sufficient to show that for any $u \in$ $H(U)$ and any neighborhood $N$ of $u$ there is a $v \in N \backslash G_{i j}$. We may assume that $N$ is of the form

$$
N=\left\{v \in H(U):\|u-v\|_{K}<\epsilon\right\},
$$


where $K$ is some compact subset of $U$ and $\epsilon$ is some positive number. We may also assume that $K=\hat{K}_{U}$. Let $\left|x-p_{i}\right|<1 / j$ be in $U \backslash K$. By the Cauchy-Kovalevskaya Theorem $H(\{x\}) \neq\{0\}$, and so by the Lax-Malgrange Theorem there is a $v \in H(U)$ such that $\|u-v\|_{K}<\epsilon$ and $|v(x)|>j$. This completes the proof of our assertion.

The situation is dramatically different when $n=1$.

Extendibility [In, p. 73]. If $a_{m}(x) \neq 0$ in $\Omega$ then any solution of (5) on an open subinterval I of $\Omega$ extends uniquely to a solution on all of $\Omega$.

From this it follows that both the uniqueness property and the Runge property hold for solutions of (5) with $q=0$. Indeed, if $K$ is a compact subset of the open interval $I$ then $K=\hat{K}_{I}$ if and only if $K$ consists of just one component. In this case not only can we find a global solution $v$ on $\Omega$ which approximates a solution $u$ given on $I$, we may even assume that $\left.v\right|_{I}=u$.

Strong Maximum Principle. Second order equations with $a_{2}(x) \neq 0$ in $\Omega$ and $a_{0}=0$ satisfy the strong maximum principle.

The proof of this is the same as the proof of [GT, Corollary 3.2], but uses Theorem 1 in [PW].

Applying the results of $\S 1$, we get the following analogs of Theorems 2.1-2.2 for solutions of second order ordinary differential equations on intervals of $\mathbb{R}^{1}$.

Theorem 3.1. Let $f$ be a continuous function on the compact, nondegenerate interval $[a, b] \subset(\alpha, \beta)$, and let $\mathscr{H}$ be the sheaf of solutions on $(\alpha, \beta)$ to

$$
P u=a_{2}(x) \frac{d^{2} u}{d x^{2}}+a_{1}(x) \frac{d u}{d x}=0,
$$

where $a_{2}$ does not vanish in $(\alpha, \beta)$. Let $h$ be an element of $\mathscr{H}([a, b])$ and suppose that there are three points $a \leq x_{1} \leq x_{2} \leq x_{3} \leq b$ such that $f\left(x_{1}\right)-$ $h\left(x_{1}\right)=f\left(x_{3}\right)-h\left(x_{3}\right)=\epsilon\|f-h\|_{[a, b]}$ and $f\left(x_{2}\right)-h\left(x_{2}\right)=-\epsilon\|f-h\|_{[a, b]}$, $\epsilon= \pm 1$. Then $h$ is a best approximation to $f$ from $\mathscr{H}([a, b])$.

Theorem 3.2. Under the conditions of Theorem $3.1 h$ is unique.

We note that under the assumption $a_{2} \neq 0$ in $\Omega$, the solution space of

$$
P u=a_{2}(x) \frac{d^{2} u}{d x^{2}}+a_{1}(x) \frac{d u}{d x}=0
$$

on an open subinterval $I$ has a basis consisting of the constant 1 and a strictly increasing function. The fact that such a basis is a Chebyshev system [KS] may also be used to show the validity of Theorems 3.1 and 3.2. As for an analog to Theorem 2.3, we remark that when $n=1$, conditions (1) and (2) are equivalent for any two compact subsets of $I$. Thus, while the corresponding statement is true, it is not particularly interesting.

One condition that does ensure the existence of an "alternant" $\left\{x_{1}, x_{2}, x_{3}\right\}$ as in Theorem 3.1 is the existence, on each open subinterval $I$, of solutions $u_{0}$ and $u_{1}$ such that $u_{0}$ is positive and $u_{1} / u_{0}$ is strictly increasing. We call $\left\{u_{0}, u_{1}\right\}$ a complete Chebyshev system. For any $\xi \in I$ there is an element in the span of $\left\{u_{0}, u_{1}\right\}$ that is negative to the left of $\xi$ and positive to the right of $\xi$. Thus, if no such alternant were to exist then we could construct a solution that is positive on $K_{+}(f-h)$ and negative on $K_{-}(f-h)$. By Kolmogorov's 
criterion, $h$ then fails to be a best approximation to $f$. Examples of differential equations whose solution space is spanned by a complete Chebyshev system are given in [KS].

\section{APPROXIMATION BY HARMONIC FUNCTIONS}

In this section we specialize our results to harmonic functions and give several examples. We start with a generalization of Theorems 0.1 and 0.2 . Let $E$ be a compact set in $\mathbb{R}^{n}$ and let $G$ be an open subset of $E$. Define $A(G, E)=$ $C(E) \cap H(G)$. Important special cases are $A(E)=A\left(E^{\circ}, E\right)$ and $A(G, \bar{G})$, which is equal to $A(\bar{G})$ when $G=\bar{G}^{\circ}$.

Theorem 4.1. Let $f \in C(E)$ and $h \in A(G, E)$ be given, and let $K_{-}=K_{-}(f-h)$ and $K_{+}=K_{+}(f-h)$. If $\hat{K}_{+} \cap \hat{K}_{-} \neq \varnothing$ (hulls relative to $\left.G\right)$, then $h$ is a best approximation to $f$ from $A(G, E)$. Conversely, if $G=\bar{G}^{\circ}$ and $h$ is a best harmonic approximation to $f$, then $\hat{K}_{+} \cap \hat{K}_{-} \neq \varnothing$, provided that $\left(K_{+} \cup K_{-}\right) \hat{=} \hat{K}_{+} \cup \hat{K}_{-}$.

Proof. The proof of sufficiency is identical to the proof of Theorem 1.1, with $\mathscr{H}(E)$ replaced by $A(G, E)$ and $E^{\circ}$ replaced by $G$. To prove necessity, suppose that $\hat{K}_{+}$and $\hat{K}_{-}$are disjoint. We first apply Lemma 1.3 with $\bar{G}$ in place of $E$ and $G$ in place of $E^{\circ}$ (recall $G=\bar{G}^{\circ}$ ) and conclude that every hollow of $\left(\hat{K}_{+} \cup \hat{K}_{-}\right) \cap \bar{G}$ meets $\mathbb{R}^{n} \backslash \bar{G}$. Since the sheaf on $\mathbb{R}^{n}$ of harmonic functions has the Runge property, there is an element $u$ of $H(\bar{G})$ that is positive on $K_{+} \cap \bar{G}$ and negative on $K_{-} \cap \bar{G}$. Using an elementary topological argument we can extend $u$ to an element $v \in A(G, E)$ such that $v>0$ on $K_{+}$and $v<0$ on $K_{-}$. As in Theorem 1.4, this implies that $h$ is not a best approximation to $f$.

Note that there is no uniqueness in Theorem 4.1 except in special cases, e.g., if $E^{\circ}=\varnothing$, or if $E^{\circ}$ is connected and $E=\overline{E^{\circ}}$ (see Example 4, below). Obviously, if $G$ is connected then $h \mid \bar{G}$ is uniquely determined.

Of course, the sheaf of harmonic functions on a given open subset of $\mathbb{R}^{n}$ satisfies the weak and strong maximum principles, and has the local positivity property, the uniqueness property, and the Runge property. Therefore, Theorems $2.1-2.3$ are valid.

In order to elucidate a connection between approximation by elements of $H(E)$ and approximation by elements of $A(E)$, we introduce the notion of fine topology. The fine topology in $\mathbb{R}^{n}[\mathrm{He}, \mathrm{La}]$ is the weakest topology making all superharmonic functions continuous (in the extended sense). This topology is finer than the usual Euclidean topology on $\mathbb{R}^{n}$. A set $E$ is called thin at a point $x$ if $x$ is not a fine limit point of $E$. In particular, $E$ is thin at $x$ whenever $x$ is not a limit point of $E$ in the Euclidean topology.

Regularity for the Dirichlet problem may be characterized in terms of the fine topology. Indeed, if $\Omega$ is a bounded open set then a point $x \in \partial \Omega$ is regular if and only if $\mathbb{R}^{n} \backslash \Omega$ is not thin at $x$.

Let $E \subset \mathbb{R}^{n}$ be a compact set. By a theorem of Keldyš every element of $A(E)$ is the uniform limit on $E$ of elements of $H(E)$ if and only if $E^{c}$ and $\left(E^{\circ}\right)^{c}$ are thin at the same points. Thus (see [La, p. 333]), if $E^{c}$ is not thin at any points of $\partial E$, then $E^{\circ}$ is regular for the Dirichlet problem and every function in $A(E)$ is the uniform limit on $E$ of elements of $H(E)$. This implies that, 
for $f$ given on such an $E$,

$$
\inf \left\{\|f-u\|_{\infty}: u \in A(E)\right\}=\inf \left\{\|f-u\|_{\infty}: u \in H(E)\right\} .
$$

Naturally, in this case $A(E)$ is just the set of solutions to the Dirichlet problem on $E^{\circ}$ with continuous data on $\partial E$.

In $\mathbb{R}^{2}$ somewhat more can be said. If $E$ is a compact subset of an open set $\Omega \subset \mathbb{R}^{2}$ then $\mathbb{R}^{2} \backslash \hat{E}_{\Omega}$ is not thin on $\partial \hat{E}_{\Omega}$. Now suppose that $\partial E=\partial \hat{E}_{\Omega}$ and let $f \in A(E)$. Then $f$ may be extended to an element $\hat{f} \in A\left(\hat{E}_{\Omega}\right)$. In [BG] it is shown that if every element of $A(E)$ has an extension to an element of $A\left(\hat{E}_{\Omega}\right)$ then every element of $A(E)$ is the uniform limit on $E$ of elements of $H(\Omega)$. In particular, it follows that if $E=\hat{E}_{\Omega}$ then every element of $A(E)$ is the uniform limit on $E$ of elements of $H(\Omega)$. Thus, for $f$ given on such an $E$,

$$
\inf \left\{\|f-u\|_{\infty}: u \in A(E)\right\}=\inf \left\{\|f-u\|_{\infty}: u \in H(\Omega)\right\} .
$$

This analysis may be used to get a different necessary condition than that in Theorem 4.1. Indeed, let $f, h, K_{+}$, and $K_{-}$be as in Theorem 4.1, let $K:=K_{+} \cup K_{-}$, and suppose that every function in $A(K)$ has an extension to a function in $A(\hat{K})$. For instance, when $n=2$, this will be the case if $\partial K=\partial \hat{K}$ [BG]. We may then proceed as in the proof of Theorem 1.4 except that we now approximate the extension to $\hat{K}$ of the function equal to, say, 1 on $K_{+}$and -1 on $K_{-}$by elements of $H(\Omega)$, to conclude that $h$ is not a best approximation to $f$. One could apply this argument, for example, if $E$ were an annulus in $\mathbb{R}^{2}$ and $K_{+}$and $K_{-}$were the inner and outer boundaries of $E$, respectively.

Example 1. Suppose that $f(x, y)$ is continuous in the plane, depends only on the distance $\rho$ from the origin and increases from 0 to 1 as $\rho$ increases from 0 to 1 . Then our theorem shows that $h \equiv 1 / 2$ is the unique best harmonic approximation to $f$ in the unit disk.

Example 2. Suppose that $f(x, y)$ is continuous in the plane, depends only on $x$, and increases from -1 to 1 as $x$ increases from -1 to 1 . Consider the firstdegree polynomial $h_{0}(x)$ of best approximation to $f(x, 0)$ on $[-1,1]$. Note that in $\mathbb{R}^{1}, h_{0}$ is the best harmonic approximation to $f(x, 0)$ on $[-1,1]$. Extend $h_{0}$ to the plane by setting $h(x, y)=h_{0}(x)$. Then $h$ will not be a best harmonic to $f$ on the unit disk, which can be seen from Theorem 2.3. We thank the referee for this example.

The following example shows that continuous functions on a compact set need not have a continuous best harmonic approximation (this is a variant of [HKL, Example 4.3]).

Example 3. Let $D$ be the unit disk in $\mathbb{R}^{2}$. Our approximating class is the set $A(\bar{D})$ of functions harmonic in $D$ and continuous in $\bar{D}$, with norm $\|\cdot\|_{\bar{D}}$.

Let $f$ be a continuous, odd function on $[-1,1]$ with $f(-1)=f(1)=0$, $f \not \equiv 0$. Extend $f$ to all of $\bar{D}$ by first setting $f=0$ on the circumference $\left\{(x, y): x^{2}+y^{2}=1\right\}$ and then solving the Dirichlet problem in both the upper half-disk $D_{+}$and the lower half-disk $D_{-}$. The resulting function, which we still denote by $f$, is harmonic in $D_{+}$and $D_{-}$, and is symmetric with respect to the $x$-axis and antisymmetric (odd) with respect to the $y$-axis in $D$. 
If $f$ has a continuous best harmonic approximation $h$, then it is unique in $A(\bar{D})$. Consequently, $h$ is also symmetric with respect to the $x$-axis and antisymmetric with respect to the $y$-axis. Since $f-h$ is harmonic in $D_{+}$and in $D_{-}$, it has no interior extrema, and thus all extrema must lie on either the circumference of $D$ or in the interval $(-1,1)$. By the characterization theorem, $\hat{K}_{+} \cap \hat{K}_{-} \neq \varnothing$, hence there is a point of $K_{+}$inside a precompact component of $D \backslash K_{-}$, or vice versa. But this is possible only if the circumference of $D$ is wholly contained in either $K_{+}$or $K_{-}$. However, this contradicts the antisymmetry of $f-h$.

Example 4. Let $E=\{|z| \leq 1\} \cup[1,2]$. Let $f$ be a continuous function on $E$ with $f(z)=f(|z|), f(|z|) \uparrow, f(0)=0$, and $f(x)=2$ for $x \in[1,2]$. If $h \in A(E)$ with $h \equiv 1$ for $|z| \leq 1$ and $1 \leq h(x) \leq 2$ for $x \in[1,2]$, then $h$ is a best approximation to $f$ from $A(E)$. Clearly there are many such functions, and so uniqueness fails, despite the fact that $\mathbb{C} \backslash E$ is connected and $\hat{K}_{+} \cap \hat{K}_{-} \neq \varnothing$.

\section{APPENDIX}

Let $\Omega_{1} \subset \Omega_{2}$ be open subsets of $\Omega$. The Lax-Malgrange property as we have stated it requires that the set difference $\Omega_{2} \backslash \Omega_{1}$ have no compact components. However, in some references, such as [Hö, p. 112], this condition is replaced by slightly different ones. In this appendix, we show the equivalence of two such conditions to the Lax-Malgrange property.

Theorem A. The following conditions are equivalent.

(a) $\Omega_{2} \backslash \Omega_{1}$ has no compact components.

(b) $\Omega_{2} \backslash \Omega_{1}$ cannot be written as the disjoint union of two sets $F$ and $L$, where $F$ is closed in $\Omega_{2}$ and $L$ is compact and nonempty.

(c) $\Omega_{2}^{*} \backslash \Omega_{1}$ is connected ( $\Omega_{2}^{*}$ is the one-point compactification of $\Omega_{2}$ ).

Proof. Suppose that (b) fails and let $F$ and $L$ be as in (b). Let $*$ be the ideal point in the one-point compactification of $\Omega_{2}$. Then $\Omega_{2}^{*} \backslash \Omega_{1}$ is the disjoint union of the two nonempty relatively open sets $F \cup\{*\}$ and $L$. Thus, $\Omega_{2}^{*} \backslash \Omega_{1}$ is not connected, i.e., (c) implies (b).

We now show that (a) implies (c). Suppose that (a) holds and suppose that $\Omega_{2}^{*} \backslash \Omega_{1}$ is the disjoint union of two relatively open-closed sets $U_{*}$ and $U_{0}$, where $U_{*}$ contains the ideal point $*$. Suppose that $U_{0}$ is nonempty, say, $x \in U_{0}$. Let $C$ be the component of $\Omega_{2} \backslash \Omega_{1}$ containing $x$. Then $C$ is contained in $U_{0}$ since, otherwise, $U_{*} \backslash\{*\}$ and $U_{0}$ separate $C$. Since $C$ is relatively closed in $\Omega_{2} \backslash \Omega_{1}$ and $U_{0}$ is compact (being the complement of an open neighborhood of $*$ ), it follows that $C$ is also compact. This contradicts (a), and so $U_{0}$ is empty, which shows that (c) holds.

There remains to show that (b) implies (a). Suppose that $\Omega_{2} \backslash \Omega_{1}$ has a compact component $K$. Let $\left\{U_{n}\right\}$ be a sequence of open subsets of $\Omega_{1}$ such that, for each $n, U_{n} \subset \bar{U}_{n} \subset U_{n+1}$ and $\cup U_{n}=\Omega_{1}$. Let $V_{n}$ denote the component of $\left(\bar{U}_{n}\right)^{c}$ containing $K$. Thus, $\left\{V_{n}\right\}$ is a nested sequence of open connected sets. We claim that $V_{n}$ is eventually compactly contained in $\Omega_{2}$. If not, then, fixing some point $p$ in $K$ and an open neighborhood $G$ of $K$, precompact in $\Omega_{2}$, we may for each $n$ construct an arc $S_{n}$ in $V_{n}$ whose initial 
point is $p$ and which lies in $G$ except for its terminal $p_{n}$ which lies on the boundary of $G$. Let $s_{n}=S_{n} \backslash\left\{p_{n}\right\}$. Label $s=\lim \sup \left\{s_{n}\right\}$, the set of all points in $\bar{G}$ each of whose neighborhoods meets infinitely many $s_{n}$. By [HY, p. 101], $s$ is a continuum in $\Omega_{2} \backslash \Omega_{1}$ which meets $K$, and hence the union of $K$ and $s$ is connected. Since $s$ also meets the boundary of $G$, the union of $K$ and $s$ strictly contains $K$. This contradicts the fact that $K$ is a component of $\Omega_{2} \backslash \Omega_{1}$. The preceding argument shows that we may assume the $V_{n}$ are eventually contained in $G$. Thus, we may fix some $V=V_{n}$ which is contained in $G$. Let $F$ be that portion of $\Omega_{2} \backslash \Omega_{1}$ which lies outside of $V$ and let $L$ be that portion of $\Omega_{2} \backslash \Omega_{1}$ which lies inside of $V$. Now $F$ is relatively closed in $\Omega_{2} \backslash \Omega_{1}$, and the same is true of $L$ since $\partial V \subset \Omega_{1}$. It follows that $L$ is closed in $\Omega_{2}$ and since $L$ is contained in the compact set $\bar{G}, L$ is itself compact. Since $F$ is closed in $\Omega_{2}$ and $L$ is compact and nonempty, (b) is violated. The proof is complete.

\section{REFERENCES}

[Ag] S. Agmon, Unicité et convexité dans les problèmes différentiels, Presses Univ. Montréal, Montréal, 1966.

[BG] T. Bagby and P. M. Gauthier, Approximation by harmonic functions on closed subsets of Riemann surfaces, J. Analyse Math. 51 (1988), 259-284.

[Bu] H. G. Burchard, Best uniform harmonic approximation, Approximation Theory II (G. G. Lorentz, C. K. Chui, L. L. Schumaker, eds.), Academic Press, San Diego, 1976, pp. 309-314.

[Ch] E. W. Cheney, Introduction to approximation theory, McGraw-Hill, New York, 1966.

[DGO] A. Dufresnoy, P. M. Gauthier, and W. H. Ow, Uniform approximation on closed sets by solutions of elliptic partial differential equations, Complex Variables Theory Appl. 6 (1986), 235-247.

[GL] N. Garofalo and F.-H. Lin, Monotonicity properties of variational integrals, $A_{p}$ weights and unique continuation, Indiana Univ. Math. J. 35 (1986), 245-268.

[GGO] P. M. Gauthier, M. Goldstein, and W. H. Ow, Uniform approximation on closed sets by harmonic functions with Newtonian singularities, J. London Math. Soc. 28 (1983), 71-82.

[GT] D. Gilbarg and N. S. Trudinger, Elliptic partial differential equations of second order, SpringerVerlag, Berlin, Heidelberg, New York, 1977.

[Ha] W. Haussmann, Approximation by harmonic functions, Topics in Multivariate Approximation (C. K. Chui, L. L. Schumaker, F. I. Utreras, eds.), Academic Press, San Diego, 1987, pp. 111-124.

[HZ] W. Haussmann and K. Zeller, $H$-sets and best uniform approximation by solutions of elliptic differential equations, Results in Math. 4 (1988), 84-92.

[HKL] W. K. Hayman, D. Kershaw, and T. J. Lyons, The best harmonic approximant to a continuous function, Anniversary Volume on Approximation Theory and Functional Analysis, ISNM 65 (P. L. Butzer, R. L. Stens, and B. Sz.-Nagy, eds.), Academic Press, San Diego, 1984, pp. 317-327.

[He] L. L. Helms, Introduction to potential theory, Wiley-Interscience, New York, 1969.

[HY] J. G. Hocking and G. S. Young, Topology, Addison-Wesley, Reading, MA, 1961.

[Hö] L. Hörmander, The analysis of linear partial differential operators. I, Springer-Verlag, Berlin, Heidelberg, New York, 1983.

[In] E. L. Ince, Ordinary differential equations, Longmans, Green, London, 1927.

[KS] S. Karlin and W. Studden, Tchebycheff systems: with applications in analysis and statistics, Wiley-Interscience, New York, 1966.

[La] N. S. Landkof, Foundations of modern potential theory, Springer-Verlag, Berlin, Heidelberg, New York, 1972. 
[Na1] R. Narasimhan, Complex analysis in one variable, Birkhäuser, Boston and Basel, 1985.

[Na2] - Analysis on real and complex manifolds, North-Holland, Amsterdam, 1968.

[PW] M. H. Protter and H. F. Weinberger, Maximum principles in differential equations, Prentice-Hall, Englewood Cliffs, NJ, 1967.

[SZ] S. Saks and A. Zygmund, Analytic functions, Monogr. Mat., Warsaw, 1952.

[Si] I. Singer, Best approximation in normed linear spaces by elements of linear subspaces, Springer-Verlag, Berlin, Heidelberg, New York, 1970.

[Wi] J. M. Wilson, A counterexample in the theory of best approximation, J. Approx. Theory (to appear).

[Zw] D. Zwick, Uniform approximation by harmonic and subharmonic functions, Approximation Theory VI (C. K. Chui, L. L. Schumaker, and J. D. Ward, eds.), Academic Press, Boston, 1989, pp. 689-692.

Département de Mathématiques et de Statistique, Université de Montréal, MonTRÉAL, QuÉbec, CANADA

E-mail address: gauthier@ere.umontreal.ca

Department of Mathematics and Statistics, University of Vermont, Burlington, VerMONT 05401-1455

E-mail address: zwick@uvm.edu 